\title{
Contamination With Fumonisin B and Deoxynivalenol Is a Threat to Egg Safety and Contributes to Gizzard Ulcerations of Newborn Chickens
}

\author{
Yihui Wang ${ }^{1,2}$, Hongkun Quan'2, Xiaohui $\mathrm{Li}^{2}$, Qiang $\mathrm{Li}^{2}$, Md Atiqul Haque ${ }^{2}$, Qin Shi' \\ Qiang $\mathrm{Fu}^{1}$ and Cheng $\mathrm{He}^{1,2 *}$ \\ ${ }^{1}$ College of Life Science and Engineering, Foshan University, Foshan, China, ${ }^{2}$ Key Lab of Animal Epidemiology \\ and Zoonoses, College of Veterinary Medicine, China Agricultural University, Beijing, China
}

OPEN ACCESS

Edited by:

Xiulan Sun,

Jiangnan University, China

Reviewed by:

Imourana Alassane-Kpembi,

Université de Montréal, Canada

Fuguo Xing,

Institute of Food Science and Technology, Chinese Academy of Agricultural Sciences (CAAS),

China

${ }^{*}$ Correspondence:

Cheng $\mathrm{He}$

hecheng@cau.edu.cn

Specialty section:

This article was submitted to

Food Microbiology,

a section of the journal

Frontiers in Microbiology

Received: 05 March 2021

Accepted: 11 June 2021

Published: 12 July 2021

Citation:

Wang Y, Quan H, Li X, Li Q, Haque MA, Shi Q, Fu Q and He C (2021) Contamination With Fumonisin

$B$ and Deoxynivalenol Is a Threat

to Egg Safety and Contributes

to Gizzard Ulcerations of Newborn

Chickens.

Front. Microbiol. 12:676671 doi: 10.3389/fmicb.2021.676671
Fumonisin B (FB) and other fumonisins, deoxynivalenol (DON), and zearalenone (ZEN) are mycotoxins (secondary metabolites of fungi) present at high levels of contamination in poultry diets and threatening the sustainability of the poultry industry and egg safety for consumers. However, residual mycotoxins in breeder eggs and their effects on chicken progeny and gizzard ulcerations remain unclear. To unveil mycotoxin contaminations from daily diets to breeder eggs, 293 poultry feed samples were collected from three large-scale poultry provinces across Northern China to Southern China. Average levels of $1,628 \pm 4.36 \mu \mathrm{g} / \mathrm{kg}$ of $\mathrm{FB}_{1}, 593 \pm 11.16 \mu \mathrm{g} / \mathrm{kg}$ of DON, $69 \pm 9.21 \mu \mathrm{g} / \mathrm{kg}$ of ZEN, $52 \pm 7.33 \mu \mathrm{g} / \mathrm{kg}$ of OTA, and $24 \pm 5.85 \mu \mathrm{g} / \mathrm{kg}$ of AFB 1 were found in feedstuffs and poultry diets using commercial ELISA kits. In terms of residual mycotoxins in breeder eggs, $\mathrm{FB}_{1}$ and $\mathrm{DON}$ contaminations dominated residues in egg albumen and yolk samples. Out of 221 breeder eggs, the average residual of $\mathrm{FB}_{1}$ in albumen were $320.6 \pm 10.12 \mu \mathrm{g} / \mathrm{kg}$ (Hebei), $420.2 \pm 10.98 \mu \mathrm{g} / \mathrm{kg}$ (Guangdong), and $549.4 \pm 10.27$ (Guangxi). Moreover, higher residual of DONs were determined in Guangdong and Guangxi provinces compared to Hebei province. ZEN, ochratoxins A (OTA), and aflatoxin $\mathrm{B}_{1}\left(\mathrm{AFB}_{1}\right)$ contamination at low levels were found in the above samples collected from afronmentioned three provinces. Based on residual mycotoxins in breeder eggs, SPF embryonated eggs aged 11 days were inoculated into albumen with different doses of $\mathrm{FB}_{1}, \mathrm{FB}_{2}$ or $\mathrm{DON}$, or a combination of $\mathrm{FB}_{1}$ and $\mathrm{DON}$, or a combination of $\mathrm{FB}_{1}$ with $\mathrm{FB}_{2}$ and $\mathrm{FB}_{3}$. $A$ lower hatching rate was observed in the chicken progenies with the combination of $24 \mu \mathrm{g}$ of $\mathrm{FB}_{1}$ and $0.1 \mu \mathrm{g}$ of DON compared to other treatments. Moreover, typical gizzard ulcerations with hemorrhagic lungs were observed in the progeny of breeder eggs post-inoculation of $24 \mu \mathrm{g}$ of $\mathrm{FB}_{1}$ and synergetic inoculation of $\mathrm{FB}_{1}$ and $\mathrm{DON}$. Finally, residual FB mycotoxins were detected in the gizzards and in the lungs of the progenies. Based on the above evidence, feed-borne FB 1 and DON are dominant mycotoxins in breeder eggs and threatening food security using breeder eggs as a Trojan horse. More importantly, the residual of FB 1 alone and in combination with of DON contamination are associated with low hatching rate and gizzard ulcerations in chicken progenies, hampering sustainable development perspectives of the poultry industry. 


\section{INTRODUCTION}

Avian gizzard ulceration has been documented frequently in the poultry industry, contributing to consistent diarrhea, poor feed conversion, and low economic benefits. Gizzard ulceration is associated with congenital factors, starvation, feed structure, nutritional deficiencies, toxic substances, infections, and microbial colonization; however, the potential mechanism remains unclear, threatening the sustainability of the poultry industry (Atiqul Haque et al., 2020).

At present, the hatching rate is less than $86.5-90.8 \%$ during the summer season in Chinese poultry farms, equal to an average of $9.5-13.5 \%$ hatching reduction in comparison with the cold season. More importantly, gizzard ulcerations are prevalent in newly hatched chickens among fast-growing broilers, slow-growing broilers (yellow broilers), and ducklings. Consequently, newborn chickens and ducklings are suffering from an average $15 \%$ mortality due to severe diarrhea and maldigestion in the first week. Unfortunately, no practical control measure is available due to elusive pathogenesis. In a previous study, adenovirus serotype 4 was identified from hemorrhagic ventricular gastric lesions in broiler flocks in South Korea, Japan, and Italy (Manarolla et al., 2009). Subsequently, Clostridium perfringens (C. perfringens) was isolated from gizzard ulceration in commercial chickens. C. perfringens is more than an opportunistic pathogen occupying a new niche following the appearance of gizzard lesions. Bacillus cereus (B.cereus), as a primary or permanent latent infection, induced gizzard ulceration and lung inflammation in SPF chickens and aggravated susceptibility to avian influenza virus H9N2 (Zhang et al., 2019). Chickens' exposure to B. cereus intensifies gizzard ulceration and pneumonia after chlamydial infection, leading to respiratory stress and breathing dysfunction (Zuo et al., 2020).

Recently, increasing levels of mycotoxins have been found in poultry and livestock diets. Mycotoxin contamination is particularly present in the hot season with high humidity. In previous reports, a study in Kenya showed that the final flour was less contaminated, while mycotoxins of deoxynivalenol (DON) and zearalenone (ZEN) were detected on the surface of the granules at high levels while temperature and time can affect the mycotoxin content of the final product (Agriopoulou et al., 2020). Dysfunctions of the liver and kidney were exacerbated in the layers after intravenous injection of $\mathrm{ZEN}, \mathrm{DON}$, and fumonisin $B_{1}\left(F_{1}\right)$ (Dassi et al., 2018). Numerous reports have documented the association between feed-borne mycotoxin and gastrointestinal tract (GIT) issues in broilers and growing pigs. Mycotoxins reduce broiler feed intake by $12 \%$ and weight gain by $14 \%$, with aflatoxin $\mathrm{B}_{1}\left(\mathrm{AFB}_{1}\right)$, ochratoxins $\mathrm{A}$ (OTA), and deoxynivalenol (DON) having the greatest effect on these parameters. The GIT is the initial site for interaction of ingested mycotoxins in the animal, and mycotoxins have a negative effect on the viability of the intestinal cells (Broom, 2015). After feeding with $\mathrm{FB}_{1}$ contaminated diets, villus height and crypt depth of the ileum were significantly reduced in broilers, and a higher percentage of birds developed subclinical necrotic enteritis in the groups fed the $\mathrm{FB}_{1}$-contaminated feed as compared to the control group, after the C. perfringens challenge (Antonissen et al., 2015).
Generally, egg albumen contains about $10.5 \%$ protein and $88.5 \%$ water, while the yolk only contains $50 \%$ water in the eggs of domestic fowl. This difference might be necessary to ensure that eggs are provisioned with adequate levels of nutrients for incubation and embryo development (Vesely et al., 1982). However, no report on correlation between mycotoxin contamination and gizzard ulcerations of chicken progenies has been recorded. Therefore, our hypothesis is that breeder eggs are highly contaminated with mycotoxins via the feed-egg chain, and synergistic mycotoxins might contribute to gastric lesions of new hatching chickens during embryonic development. We collected feed samples and breeder eggs from Northern China to Southern China. Afterward, SPF embryonated chickens, aged 11 days, were inoculated artificially with $\mathrm{FB}_{1}, \mathrm{FB}_{2}$ or $\mathrm{DON}$, or a combination of $\mathrm{FB}_{1}$ with $\mathrm{DON}$ or synergetic inoculation of $\mathrm{FB}_{1}$ with $\mathrm{FB}_{2}$ and $\mathrm{FB}_{3}$. The hatching rate, lesions, and mycotoxin residues were monitored in lungs and gastric system on hatching day.

\section{MATERIALS AND METHODS}

\section{Determination of Mycotoxins in Poultry Feed and Breeder Eggs}

A cross-sectional survey was carried out to collect feed samples from three provinces in China-Hebei, Guangdong, and Guangxi-in September 2017, representing a large poultry population both in Northern China and in Southern China. A total of 293 feed samples and 221 breeder eggs were collected from the aforementioned poultry farms, namely, 32 corns, 20 soybeans, 19 brans, 19 choline chlorides, 20 feed additives, 95 start diets, and 88 finished diets. As for breeder eggs, 72, 80, and 69 eggs were collected from Hebei, Guangxi and Guangdong in the same poultry farms, respectively.

Regarding determination of mycotoxins, poultry feedstuff and diets were treated and detected using commercial kits (Beacon Analytical Systems Inc., TX, United States). For measurement of the mycotoxin residues in breeder eggs, the protocols were modified as follows. Firstly, the albumen and yolk were separated using a manual egg separator. Afterward, the egg samples were lyophilized for $24 \mathrm{~h}$ to prepare powder form (Thermo Fisher Scientific Inc., Shanghai, China) and kept at $-80^{\circ} \mathrm{C}$ until use. Finally, quantities of FB, DON and ZEN were determined using ELISA kits (Beacon Analytical Systems Inc., United States).

\section{Effect of Mycotoxins on the Hatching Rate and Gizzard Ulceration of Embryonated Chickens}

$\mathrm{FB}_{1}, \mathrm{FB}_{2}$, and $\mathrm{FB}_{3}$ samples (purity 98\%) were purchased from commercial products (Pribolab Pte. Ltd., Singapore, Singapore). SPF embryonated chickens, aged 11 days, were purchased from a commercial company (Boehringer Ingelheim Inc., Beijing, China). The experimental protocols were approved by an Ethical Reviewing Board at China Agricultural University (Approval code: IACUC201700802), based on guidelines from the Institutional Animal Care and Use Committee (IACUC). 
This follows humane protocols that minimize pain in the animals. All hatching chickens were euthanized at the end of the study in a $\mathrm{CO}_{2}$ chamber using $100 \% \quad \mathrm{CO}_{2}$ at a flow rate of $10-30 \%$ of the chamber volume per minute, and the birds were observed for the absence of breathing activities and loss of heartbeat. The $\mathrm{CO}_{2}$ flow lasted for at least $1 \mathrm{~min}$ after breathing arrest. After confirmation of death, an additional secondary physical euthanasia was carried out before tissue collection as previously described (Chu et al., 2017).

A total of 112 embryonated chickens, aged 11 days, were randomly divided into six groups, namely, four experimental groups with 24 chicken embryos in each group and two control groups with 8 chicken embryos per group. The experimental groups consisted of the $\mathrm{FB}_{1}$ group, the $\mathrm{FB}_{2}$ group, the $\mathrm{FB}_{1}+\mathrm{FB}_{2}+\mathrm{FB}_{3}$ group, and the $\mathrm{FB}_{1}+\mathrm{DON}$ group, while each experimental group was divided into three different doses, the high-dose group, the moderate-dose group and the lowdose group, with eight chicken embryos per group. Meanwhile, the control groups included the DON control group and the methanol reagent control group, with eight chicken embryos per group. The chicken embryos received $0.1 \mu \mathrm{g}$ of DON or $0.1 \mathrm{ml}$ of methanol as the control group. Briefly, $\mathrm{FB}_{1}$ samples were prepared by adding $64 \mathrm{mg}$ of the toxin to $10 \mathrm{ml}$ solutions containing methanol:water (1:1 vol/vol). The $\mathrm{FB}_{2}$ and $\mathrm{FB}_{3}$ samples were prepared by dissolving $8 \mathrm{mg}$ of the toxins in $5 \mathrm{ml}$ solutions with methanol:water (1:1 vol/vol). The $\mathrm{FB}_{1}+\mathrm{FB}_{2}+\mathrm{FB}_{3}$ stock samples included three ratios of toxins (3:1:1 weight ratio), by adding $9.6 \mathrm{mg}$ of $\mathrm{FB}_{1}, 3.2 \mathrm{mg}$ of $\mathrm{FB}_{2}$, and $3.2 \mathrm{mg}$ of $\mathrm{FB}_{3}$ to $10 \mathrm{ml}$ solutions containing methanol:water (1:1 vol/vol). Afterward, serial dilutions were prepared as described in Table 1. Finally, the FB1 + DON samples were prepared as described above (Henry and Wyatt, 2001).

Prior to inoculation into the SPF chicken albumen, poorly growing embryonated eggs aged 11 days were eliminated from the experiment (Willems et al., 2014). Before inoculation, the embryonated eggs were sterilized with 1\% iodine and $75 \%$ alcohol, and then $100 \mu \mathrm{l}$ preparations per egg were injected into the embryo albumen. Post inoculation, the injected hole was sealed with wax and embryonated eggs were incubated at $37^{\circ} \mathrm{C}$ and monitored daily for mortality, development, and hatching numbers.

\section{Determination of Mycotoxins in the Gizzard and Lungs of Newborn Chickens}

\section{FB Detection}

After hatching at day 20, the newborn chickens were euthanized by $\mathrm{CO}_{2}$ flowing chamber, as per the above protocol. Postmortem, the gizzards and lungs of the newborn chicks were collected aseptically, the organs were homogenized and $1 \mathrm{~g}$ of tissue was blended with $10 \mathrm{ml}$ of $70 \%$ methanol/ultra-water. Subsequently, the samples were centrifugated at $12,000 \mathrm{rpm}$ for $5 \mathrm{~min}$, and $1 \mathrm{ml}$ of the supernatants was collected and mixed with $2 \mathrm{ml}$ of $n$-hexane. Afterward, the samples were centrifuged to discard $n$-hexane at $12,000 \mathrm{rpm}$ for 5 mins. Finally, $100 \mu \mathrm{l}$ of the
TABLE 1 | Experimental designs with mycotoxin alone or synergetic inoculations.

\begin{tabular}{lcc}
\hline Groups & Toxins & Doses $(\boldsymbol{\mu} \mathbf{g} / \mathbf{e g g})$ \\
\hline 1 & $\mathrm{FB}_{1}$ & 24 \\
& & 12 \\
2 & $\mathrm{FB}_{2}$ & 48 \\
& & 24 \\
& & 12 \\
3 & $\mathrm{FB}_{1}+\mathrm{FB}_{2}+\mathrm{FB}_{3}$ & $24(14.4+4.8+4.8)$ \\
& & $12(7.2+2.4+2.4)$ \\
4 & & $6(3.6+1.2+1.2)$ \\
& & $0.1+12$ \\
5 & & $0.1+6$ \\
6 & & $0.1+3$ \\
& & 0.1 \\
& & $\mathrm{NSON}$
\end{tabular}

All embryonated eggs were randomly divided into six groups, namely, four experimental groups (each experimental group consisting of the high-dose group, the moderate-dose group, and the low-dose group, eight replicates per group) and two control groups (the DON control group and the methanol group, eight chicken embryos per group). Before inoculation, poorly growing embryonated eggs were eliminated from the experiment.

lower samples was collected and $900 \mu \mathrm{l}$ of $70 \%$ methanol/ultrawater was added, and the mixture was blended together. FB concentrations were determined using commercial ELISA kits (Beacon Analytical Systems Inc., TX, United States), as described in the protocol.

\section{DON Detection}

The sample tissues of gizzards and lungs were prepared as per the above procedures, and the pre-treatment was modified as follows. Briefly, $5 \mathrm{~g}$ of tissue was homogenized with $25 \mathrm{ml}$ of ultrapure water and then centrifuged at $12,000 \mathrm{rpm}$ for 5 mins. Afterward, $1 \mathrm{ml}$ of the supernatant was added to $2 \mathrm{ml}$ of $n$-hexane and then centrifuged at $12,000 \mathrm{rpm}$ for $5 \mathrm{mins}$. Lastly, the upper $n$-hexane was removed and $200 \mu l$ of the lower sample was blended with $800 \mu \mathrm{l}$ of ultrapure water, to be used as the final sample. DON concentrations were quantified using commercial ELISA kits (Beacon Analytical Systems Inc., TX, United States).

\section{Statistical Analysis}

Mycotoxin concentrations and lesion scores were statistically analyzed using SPSS 17.0 version to perform a one-way ANOVA with the LSD post hoc test on at least three independent replicates. $p$-values $<0.05$ were considered statistically significant for each test, and when $p<0.01$, the results were very significant. Hatching rate was statistically analyzed using SPSS 17.0 version to perform a chi-square test with categorical variable. A $p$ value of $<0.05$ was considered to be a significant difference for each test, and a $p$-value of $<0.01$ was considered to be very significant. 


\section{RESULTS}

\section{Determination of Mycotoxins in Poultry Feed and Breeder Eggs}

A total of 110 feedstuffs and 183 concentrated feed samples were included in the survey. The average concentrations were $1,628 \pm 4.4 \mu \mathrm{g} / \mathrm{kg}$ of $\mathrm{FB}, 593 \pm 11.2 \mu \mathrm{g} / \mathrm{kg}$ of $\mathrm{DON}$, $69 \pm 9.2 \mu \mathrm{g} / \mathrm{kg}$ of ZEN, $52 \pm 7.3 \mu \mathrm{g} / \mathrm{kg}$ of OTA, and $24 \pm 5.9 \mu \mathrm{g} / \mathrm{kg}$ of AFT, while the finished diet and fermented soybeans were determined to contain $3,450 \pm 2.6 \mu \mathrm{g} / \mathrm{kg}$ and $2,300 \pm 6.2 \mu \mathrm{g} / \mathrm{kg}$ of FB, respectively. As for DON contamination, the feedstuffs were found to have high contamination in the corns, brans, and feed additives, amounting to $678 \pm 5.23 \mu \mathrm{g} / \mathrm{kg}$, $813 \pm 3.3 \mu \mathrm{g} / \mathrm{kg}$, and $1200 \pm 8.2 \mu \mathrm{g} / \mathrm{kg}$, respectively. As for the AFT concentration, low contamination of both the feedstuffs and poultry diets were found in comparison with OTA and ZEN levels (Table 2). In the survey, 221 breeder eggs were collected to determine mycotoxin contamination across three provinces. As for average FB residues, $320 \pm 10.1 \mu \mathrm{g} / \mathrm{kg}, 420 \pm 10.9 \mu \mathrm{g} / \mathrm{kg}$, and $549 \pm 10.3 \mu \mathrm{g} / \mathrm{kg}$ were detected in the albumen samples, while $151 \pm 9.8 \mu \mathrm{g} / \mathrm{kg}, 321 \pm 5.1 \mu \mathrm{g} / \mathrm{kg}$, and $669 \pm 8.5 \mu \mathrm{g} / \mathrm{kg}$ were determined in yolk samples in the three provinces. Moreover, $184 \pm 9.3 \mu \mathrm{g} / \mathrm{kg}, 507 \pm 12.43 \mu \mathrm{g} / \mathrm{kg}$, and $658 \pm 2.62 \mu \mathrm{g} / \mathrm{kg}$ of DON concentrations were quantified in the albumen, which were comparable to the contamination levels in the yolk samples. High concentrations of FB and DON were detected in Guangdong and Guangxi provinces, Southern China, compared to Hebei province, except for FB residues in the albumen. Regarding ZEN, OTA, and AFT contaminations, higher residues were found in the albumens collected from Guangdong and Guangxi provinces, compared to those of Hebei province (Table 3).

\section{Effect of Mycotoxins on Hatching Rate and Gizzard Ulceration of Embryonated Chickens}

Regarding hatching rate, 88,75 , and $50 \%$ hatching rate were found in the three serial $\mathrm{FB}_{1}$ groups $(6-24 \mu \mathrm{g})$, while 75 , 50, and $37.5 \%$ hatching rate were found in the serial $\mathrm{FB}_{1}+\mathrm{DON}$ groups (from 3 to $12 \mu \mathrm{g} \mathrm{FB}_{1}$ ), indicating a dose-dependent effect. Obviously, a lower hatching rate was observed in the high $\mathrm{FB}_{1}+\mathrm{DON}$ group compared to the high $\mathrm{FB}_{1}$ group, $\mathrm{FB}_{1}+\mathrm{FB}_{2}+\mathrm{FB}_{3}$, the high $\mathrm{FB} 2$ group, and the $\mathrm{DON}$ group $(p<0.05)$. Moreover, 100,88 , and $75 \%$ hatching rate were calculated in the three serial $\mathrm{FB}_{2}$ groups (from 12 to $48 \mu \mathrm{g}$ ). However, no embryonic mortality was found in the combination of the $\mathrm{FB}_{1}+\mathrm{FB}_{2}+\mathrm{FB}_{3}$ group and the DON control group (Figure 1). Post-mortem, the gizzard lesions were shown in Figure 2, and a significant increase in gizzard ulcerations was observed in the high $\mathrm{FB}_{1}+\mathrm{DON}$ group $\left(\mathrm{FB}_{1} 12 \mu \mathrm{g}+\mathrm{DON}\right.$ $0.1 \mu \mathrm{g})$, the high $\mathrm{FB}_{1}$ group $(24 \mu \mathrm{g})$, the high $\mathrm{FB}_{2}$ group $(48 \mu \mathrm{g})$, and the combination of high $\mathrm{FB}_{1}$ with $\mathrm{FB}_{2}$ and $\mathrm{FB}_{3}\left(\mathrm{FB}_{1}\right.$ $14.4 \mu \mathrm{g}+\mathrm{FB}_{2} 4.8 \mu \mathrm{g}+\mathrm{FB}_{3} 4.8 \mu \mathrm{g}$ ), amounting to $100,85,50$, and $45 \%$ of the lesions, respectively. In the moderate groups, 75 , $63,38,25$, and $25 \%$ of the gastric lesions were found in the mixed group $\left(\mathrm{FB}_{1} 6 \mu \mathrm{g}+\mathrm{DON} 0.1 \mu \mathrm{g}\right)$, the $\mathrm{FB}_{1}(12 \mu \mathrm{g})$ group, the $\mathrm{FB}_{2}$ $(24 \mu \mathrm{g})$ group, the three $\mathrm{FB}$ synergetic groups $\left(\mathrm{FB}_{1} 7.2 \mu \mathrm{g}+\mathrm{FB}_{2}\right.$ $\left.2.4 \mu \mathrm{g}+\mathrm{FB}_{3} 2.4 \mu \mathrm{g}\right)$, and the DON group $(0.1 \mu \mathrm{g})$. Obviously, above $50 \%$ gastric lesions were found in the high $\mathrm{FB}_{1}+\mathrm{DON}$ group, the high $\mathrm{FB} 1$ group, the moderate $\mathrm{FB}_{1}+\mathrm{DON}$ group, the moderate $\mathrm{FB} 1$ group, and the low $\mathrm{FB}_{1}+\mathrm{DON}$ group. A significant increase in gastric lesions was found in both the $\mathrm{FB}_{1}+\mathrm{DON}$ group and the FB1 group compared to the other groups inoculated with the same dose $(p<0.05)$; the rate of gizzard ulceration of newborn chicken was shown in Figure 3. Regarding the combination of $\mathrm{FB}_{1}$ and $\mathrm{DON}, 37.5 \%$ hatching rate and $100 \%$ gastric lesions were observed in the high $\mathrm{FB}_{1}+\mathrm{DON}$ group, indicating that two mycotoxins yielded a synergetic effect on development of the embryonated chickens.

\section{Determination of Mycotoxins in the Gizzard and Lungs of Newborn Chickens}

Using a commercial ELISA kit, gizzard-contaminated FB mycotoxins were determined to have a dose-dependent effect in the $\mathrm{FB}_{1}+\mathrm{DON}$ group, the $\mathrm{FB}_{1}$ group, the $\mathrm{FB}_{2}$ group, the three FB combination group, and the DON group. Consequently, $260 \pm 27.3,129 \pm 21.0$, and $98 \pm 10.2 \mu \mathrm{g} / \mathrm{kg}$ of FB were detected in the gizzard tissues in the high $\mathrm{FB}_{1}+\mathrm{DON}$ group, the moderate $\mathrm{FB}_{1}+\mathrm{DON}$ group, and the low $\mathrm{FB}_{1}+\mathrm{DON}$ group, respectively. Obviously, significantly higher FB mycotoxins were found in the high $\mathrm{FB}_{1}$ group as compared to the moderate $\mathrm{FB}_{1}+\mathrm{DON}$ group $(p<0.05)$ and the low $\mathrm{FB}_{1}+\mathrm{DON}$ group $(p<0.01)$. Moreover,

TABLE 2 | Average residues of FB, DON, ZEN, OTA, and AFT in feedstuffs and feeds ( $\mu \mathrm{g} / \mathrm{kg})$.

\begin{tabular}{|c|c|c|c|c|c|c|}
\hline Sample & No. & FB & DON & ZEN & OTA & AFT \\
\hline Cardamom & 20 & $2,300 \pm 6.2^{\mathrm{c}}$ & $547 \pm 12.1^{\mathrm{a}}$ & $85 \pm 7.8^{b}$ & $89 \pm 4.3^{b}$ & $26 \pm 8.2^{a}$ \\
\hline Choline chloride & 19 & $236 \pm 4.4^{c}$ & $56 \pm 5.0^{c}$ & $56 \pm 2.1^{a}$ & $15 \pm 3.4^{b}$ & $9 \pm 7.3^{b}$ \\
\hline Feed additives & 20 & $2,145 \pm 8.3^{c}$ & $1,200 \pm 8.2^{c}$ & $100 \pm 9.5^{b}$ & $46 \pm 5.2^{\mathrm{a}}$ & $29 \pm 10.1^{a}$ \\
\hline Start diets & 95 & $567 \pm 7.0^{c}$ & $430 \pm 4.0^{b}$ & $130 \pm 4.3^{c}$ & $39 \pm 6.3^{b}$ & $31 \pm 4.6^{a}$ \\
\hline Average & & $1628 \pm 4.4$ & $593 \pm 11.2$ & $69 \pm 9.2$ & $52 \pm 7.3$ & $24 \pm 5.9$ \\
\hline
\end{tabular}

${ }^{a} p>0.05$ when compared with the average level of FB or DON, or ZEN or OTA, or AFT residue in the same column. ${ }^{b} p<0.05$ when compared with the average level of FB or DON, or ZEN or OTA, or AFT residue in the same column. ${ }^{c} p<0.01$ when compared with the average level of FB or DON, or ZEN or OTA, or AFT residue in the same column. The data were expressed as the mean $\pm S D$. 
TABLE 3 | Detection of FB, DON, ZEN, OTA, and AFT in the yolk and albumen of breeder eggs.

\begin{tabular}{|c|c|c|c|c|c|c|}
\hline \multirow[t]{2}{*}{ Sample point } & \multicolumn{2}{|c|}{ Hebei } & \multicolumn{2}{|c|}{ Guangdong } & \multicolumn{2}{|c|}{ Guangxi } \\
\hline & Albumen & Yolk & Albumen & Yolk & Albumen & Yolk \\
\hline No. & 72 & 72 & 80 & 80 & 69 & 69 \\
\hline FB & $320 \pm 10.1^{b}$ & $151 \pm 9.8^{b}$ & $420 \pm 11.0^{\mathrm{a}}$ & $321 \pm 5.1^{b}$ & $549 \pm 10.3^{b}$ & $669 \pm 8.5^{b}$ \\
\hline DON & $184 \pm 9.3^{b}$ & $192 \pm 11.7^{b}$ & $507 \pm 12.4^{b}$ & $471 \pm 6.3^{a}$ & $658 \pm 2.6^{b}$ & $515 \pm 8.0^{b}$ \\
\hline ZEN & $0.2 \pm 1.2^{\mathrm{a}}$ & $12 \pm 2.7^{a}$ & $0.7 \pm 0.8^{a}$ & $23 \pm 3.7^{b}$ & $0.3 \pm 0.5^{a}$ & $15 \pm 6.3^{a}$ \\
\hline OTA & $2.5 \pm 1.6^{a}$ & $1 \pm 0.6^{a}$ & $13.3 \pm 1.5^{b}$ & $9.7 \pm 1.3^{b}$ & $7.4 \pm 1.3^{b}$ & $5.4 \pm 1.2^{\mathrm{a}}$ \\
\hline $\mathrm{AFT}$ & $3.5 \pm 1.3^{a}$ & $0.8 \pm 0.3^{a}$ & $1 \pm 1.9^{a}$ & $0.7 \pm 1.7^{a}$ & $11.3 \pm 1.1^{b}$ & $6.2 \pm 0.9^{b}$ \\
\hline
\end{tabular}

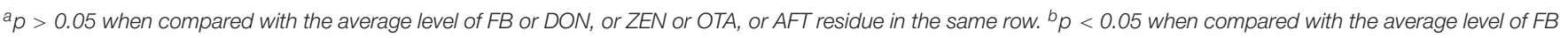
or DON, or ZEN or OTA, or AFT residue in the same row. The data were expressed as the mean $\pm S D$.

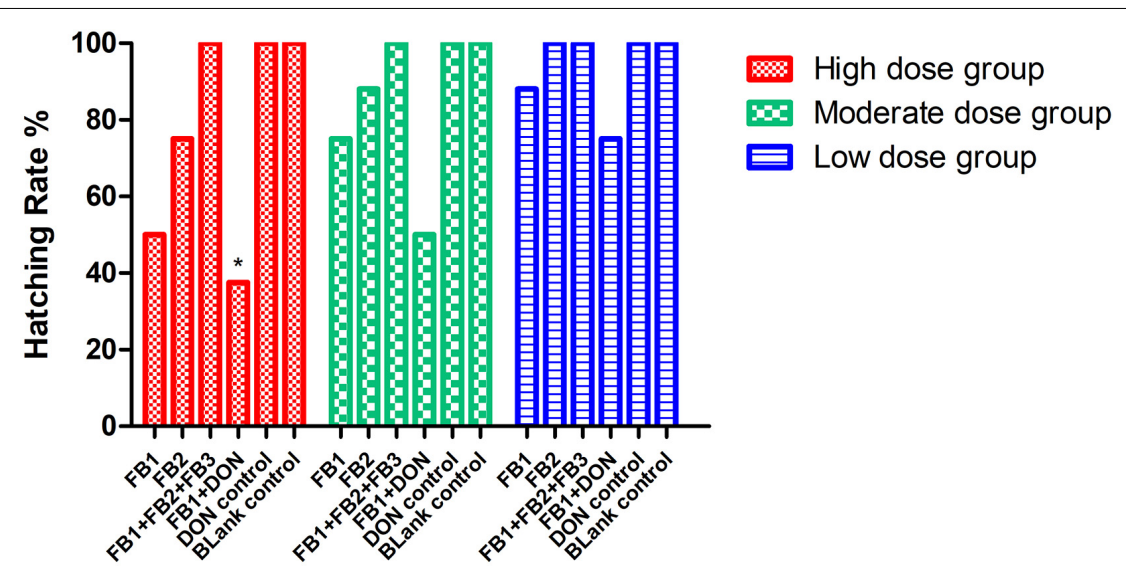

FIGURE 1 | Hatching rate post-inoculation with serial concentrations of FB or DON, or synergetic inoculation. *Indicates $p<0.05$ when the high FB $1+$ DON group was compared to the high $\mathrm{FB}_{1}$ group, the high $\mathrm{FB}_{1}+\mathrm{FB}_{2}+\mathrm{FB}_{3}$ group, and the DON group ( $p=0.037$ ), while no significant difference was found when the moderate $\mathrm{FB}_{1}+\mathrm{DON}$ group was compared to other moderate groups and the DON group $(p=0.114)$. Similarly, no statistical difference was found when the low $\mathrm{FB}_{1}+\mathrm{DON}$ group was compared to other low groups $(p=0.427)$. The data were analyzed by Chi-square test with SPSS as the categorical variable.

a significant difference was found between the high $\mathrm{FB}_{1}$ group and the low $\mathrm{FB}_{1}$ group. A similar trend was found among the $\mathrm{FB}_{2}$ groups and the $\mathrm{FB}_{1}+\mathrm{FB}_{2}+\mathrm{FB}_{3}$ groups.

On contrary to the mycotoxin residue in the gizzards, $297 \pm 20.3,170 \pm 41.2$, and $123 \pm 4.2 \mu \mathrm{g} / \mathrm{kg}$ of $\mathrm{FB}$ contents were detected in the lungs in the high $\mathrm{FB}_{1}+\mathrm{DON}$ group, the moderate $\mathrm{FB}_{1}+\mathrm{DON}$ group, and the low $\mathrm{FB}_{1}+\mathrm{DON}$ group, respectively. A statistical difference was found between the high $\mathrm{FB}_{1}+$ DON group and the low $\mathrm{FB}_{1}+$ DON group $(p<0.05)$. Regarding FB residue, a similar difference was found between the high $\mathrm{FB}_{1}$ group and the low $\mathrm{FB}_{1}$ group, and no significance was detected between the high $\mathrm{FB}_{1}$ group and the moderate $\mathrm{FB}_{1}$ group. More interestingly, a dose-dependent manner of FB residue was found in the gizzards and in the lungs post-treatment. As for DON residue, no dose effect was found in the lung tissues (Table 4).

\section{DISCUSSION}

In our survey, out of 293 feed diets, residues of FB and DON were two dominant mycotoxins both in feedstuff and concentrated poultry diets. More interestingly, high contaminations of FB and DON were found in the egg albumens collected from Southern China (Guangxi and Guangdong provinces) compared to Northern China (Hebei province). Although FB and DON contaminations were lower than the maximal tolerance limits (MTLs) of Chinese feed mycotoxins (Atiqul Haque et al., 2020), the above two egg-borne mycotoxins were equal to $10 \%$ of the residual mycotoxins in poultry feeds, indicating higher contamination in comparison with European MTLs (20-100 $\mathrm{mg} / \mathrm{kg}$ ) (Cordeiro et al., 2013). Our investigation indicated that residues of $\mathrm{FB}$ and $\mathrm{DON}$ could be absorbed into the gastrointestinal system and delivered to breeder eggs and commercial consumable eggs, threatening the development of new hatching birds and human consumers via eating eggs or egg products.

In previous reports, $\mathrm{FB}$ was the most dominant mycotoxin in maize and sorghum, at 101-1838 $\mu \mathrm{g} / \mathrm{kg}$ in maize and 81.5$361 \mu \mathrm{g} / \mathrm{kg}$ in sorghum (Warth et al., 2012). Moreover, the two mycotoxins were common contamination in feedstuffs and poultry diets during the hot season, in comparison with a low-temperature climate. Our data confirm that mycotoxin production correlates with high temperatures (Spanic et al., 2019). In our study, ZEN, OTA, and AFT contaminations were determined to be low residues in both poultry diets and breeder 


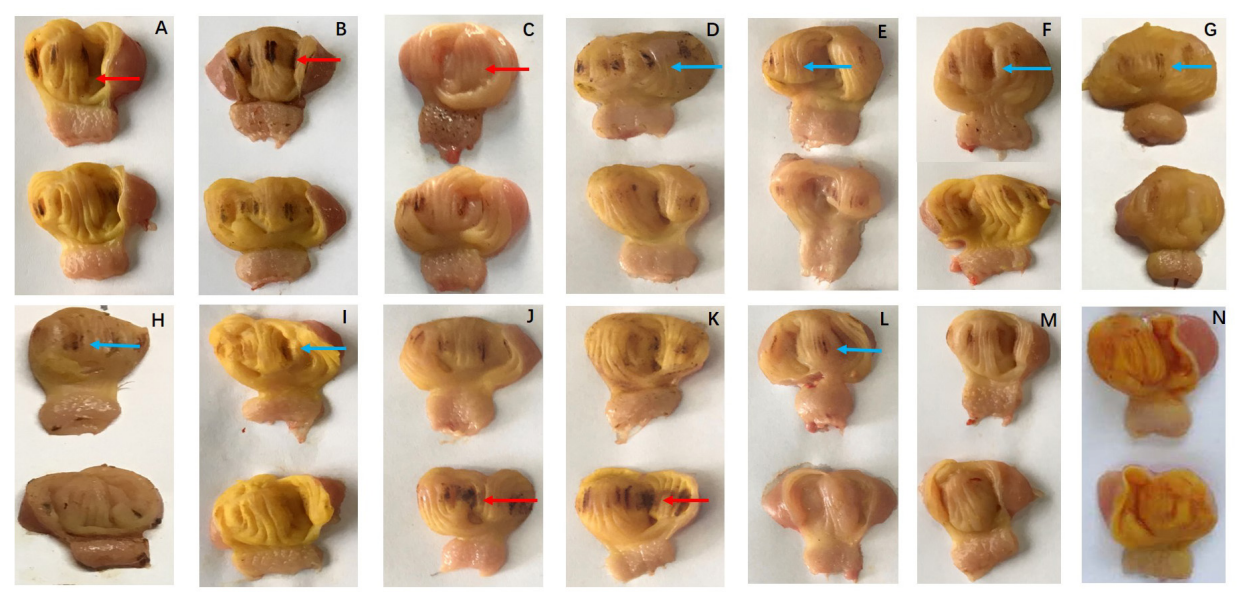

FIGURE 2 | Gizzard ulcerations of chicken progenies post-inoculation with FB or DON, or synergetic inoculation. (A) FB $6 \mu \mathrm{g} ;$ (B) FB $12 \mu \mathrm{g} ;$ (C) FB $24 \mu \mathrm{g}$; (D) FB $12 \mu \mathrm{g}$; (E) FB $24 \mu \mathrm{g}$; (F) FB $48 \mu \mathrm{g}$; (G) FB $3.6 \mu \mathrm{g}+\mathrm{FB}_{2} 1.2 \mu \mathrm{g}+\mathrm{FB}_{3} 1.2 \mu \mathrm{g}$; (H) FB $7.2 \mu \mathrm{g}+\mathrm{FB}_{2} 2.4 \mu \mathrm{g}+\mathrm{FB}_{3} 2.4 \mu \mathrm{g}$; (I) FB $14.4 \mu \mathrm{g}+\mathrm{FB}_{2}$ $4.8 \mu \mathrm{g}+\mathrm{FB}_{3} 4.8 \mu \mathrm{g}$; (J) FB $3 \mu \mathrm{g}+\mathrm{DON} 0.1 \mu \mathrm{g}$; (K) FB $6 \mu \mathrm{g}+$ DON $0.1 \mu \mathrm{g}$; (L) FB $12 \mu \mathrm{g}+$ DON $0.1 \mu \mathrm{g}$; (M) DON $0.1 \mu \mathrm{g}$; (N) Control group. Severe gastric lesions were marked with a red arrow, and moderate lesions were labeled with a blue arrow. Peeling and shedding of the gizzard membranes were evident both in the high FB1 group and the high FB $1+$ DON group. Additionally, severe hemorrhagic lesions were observed in the above two groups.

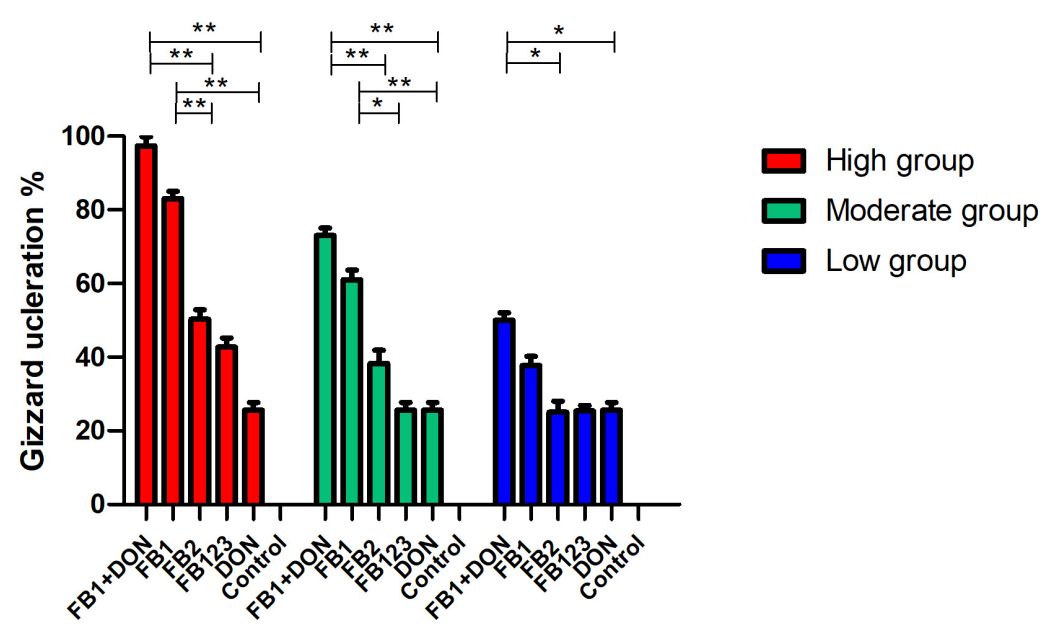

FIGURE 3 | Percentage of gizzard ulceration of chicken progenies post-inoculation with $\mathrm{FB}_{1}$ or $\mathrm{DON}$, or synergetic inoculation. Post-mortem, both the high $\mathrm{FB}_{1}+\mathrm{DON}$ group and the high $\mathrm{FB}_{1}$ group developed higher lesions of gizzard ulcerations compared to the high $\mathrm{FB}_{2}$ group, or the high $\mathrm{FB}_{1}+\mathrm{FB}_{2}+\mathrm{FB}_{3}$ group or the DON group $\left(p<0.01\right.$ ), while significant difference was found in the moderate $\mathrm{FB}_{1}+\mathrm{DON}$ group compared to the moderate $\mathrm{FB}_{2}$ group, or the moderate $\mathrm{FB}_{1}+\mathrm{FB}_{2}+\mathrm{FB}_{3}$ group or the DON group $(p<0.01)$. As for the moderate $\mathrm{FB}_{1}$ group, a statistical difference was found compared to the moderate $\mathrm{FB}_{2}$ group, the moderate $\mathrm{FB}_{1}+\mathrm{FB}_{2}+\mathrm{FB}_{3}$ group, and the DON group $(p<0.05)$. The data were expressed as the mean $\pm \mathrm{SD}$. ${ }^{*} p<0.05 ;{ }^{* *} p<0.01$.

eggs, which was different from high contaminations of OTA and AFT in Pakistan due to diverse climates (Khan et al., 2014).

There are numerous conflicts between feed-borne mycotoxins and layers' production. Regarding the egg security due to mycotoxin contaminations, limited data have been obtained on the transmission of mycotoxins from feed to eggs and meat tissues. A previous report indicated that roughly $0.31 \%$ of DON in feed went into the eggs (Prelusky et al., 1989). Laying hens were fed a diet containing DON [approximately $20 \mathrm{mg} / \mathrm{kg}(-1)$ ] and
ZEN [0.5 $\mathrm{mg} \mathrm{kg} \mathrm{(-1)],} \mathrm{and} \mathrm{no} \mathrm{adverse} \mathrm{effects} \mathrm{on} \mathrm{egg} \mathrm{production}$ were observed because the levels of DON were insignificant compared to other sources (Sypecka et al., 2004). Furthermore, low contamination of DON $(2.6-17.9 \mu \mathrm{g} / \mathrm{kg})$ and its metabolite de-epoxy-DON (DOM-1, 2.4-23.7 $\mu \mathrm{g} / \mathrm{kg}$ ) were detected in home-produced egg samples in Belgium during autumn 2006 and spring 2007, and consumption of these eggs contaminated with DON contributed to less than $1 \%$ of the provisional maximum tolerable daily intake of $1000 \mu \mathrm{g} / \mathrm{kg}$ body weight established 
TABLE 4 | Residue of FB/DON in the gizzard and lungs of newborn chickens ( $\mu \mathrm{g} / \mathrm{kg}$ ).

\begin{tabular}{|c|c|c|c|c|c|c|c|}
\hline Samples & Groups & $\mathrm{FB}_{1}+\mathrm{DON}$ & $\mathbf{F B}_{1}$ & $\mathrm{FB}_{2}$ & $\mathrm{FB}_{1}+\mathrm{FB}_{2}+\mathrm{FB}_{3}$ & DON & CONTROL \\
\hline \multirow[t]{3}{*}{ Gizzard } & High group & $260 \pm 27.3^{a} / 10.2 \pm 2.2^{a}$ & $193 \pm 15.9^{a}$ & $128 \pm 12.7^{a}$ & $221 \pm 13.0^{a}$ & No data & 0 \\
\hline & Moderate group & $129 \pm 21.0^{\mathrm{b}} / 11 \pm 1.8^{\mathrm{a}}$ & $110 \pm 13.9^{a}$ & $67 \pm 11.0^{\mathrm{b}}$ & $155 \pm 12.3^{a}$ & $10 \pm 2.8^{a}$ & 0 \\
\hline & Low group & $98 \pm 10.2^{c} / 3 \pm 2.7^{b}$ & $69 \pm 7.2^{b}$ & $45 \pm 6.5^{\mathrm{b}}$ & $110 \pm 17.9^{b}$ & No data & 0 \\
\hline \multirow[t]{3}{*}{ Lung } & High group & $297 \pm 20.3^{a} / 9 \pm 6.1^{a}$ & $216 \pm 16.2^{a}$ & $202 \pm 13.6^{a}$ & $253 \pm 15.1^{a}$ & No data & 0 \\
\hline & Moderate group & $170 \pm 41.2^{\mathrm{a} / 7} \pm 3.1^{\mathrm{a}}$ & $131 \pm 12.6^{a}$ & $83 \pm 7.9^{b}$ & $162 \pm 11.3^{a}$ & $3 \pm 1.3^{b}$ & 0 \\
\hline & Low group & $123 \pm 4.2^{b} / 10 \pm 2.4^{a}$ & $65 \pm 10.5^{b}$ & $65 \pm 1.5^{c}$ & $128 \pm 12.4^{b}$ & No data & 0 \\
\hline
\end{tabular}

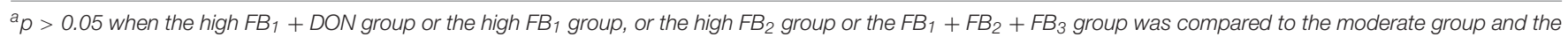
low groups in the same column.

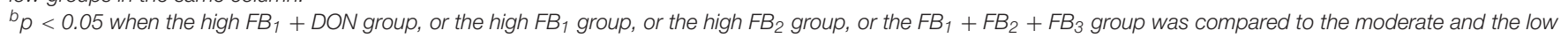
group in the same column.

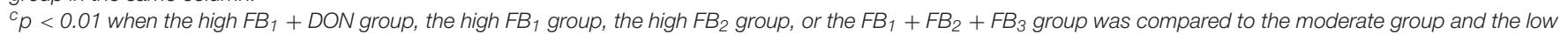
group in the same column. The data were expressed as the mean $\pm S D$.

by FAO/WHO (Tangni et al., 2009). Later on, birds received mycotoxin-contaminated diets for 6 weeks and followed by a 4-week recovery phase. Egg quality, size, and yolk weight were reduced significantly, while egg shape was ameliorated (Lee et al., 2012). More recently, out of 152 egg samples from Jiangsu (JS), Zhejiang (ZJ), and Shanghai (SH) in China, the main mycotoxins were DON, 15-AcDON, and 3-AcDON, with medians of $45.9 \mu \mathrm{g} / \mathrm{kg}, 51.5 \mu \mathrm{g} / \mathrm{kg}$, and $5.94 \mu \mathrm{g} / \mathrm{kg}$, respectively, while $0.35 \mu \mathrm{g} / \mathrm{kg}$ of ZEN metabolite ( $\beta$-ZAL) was detected, and $\mathrm{AFTB}_{1}$ was found in only one sample at a concentration of $1.46 \mu \mathrm{g} / \mathrm{kg}$. The aforementioned egg residuals were lower than DON contaminations in our survey due to the mild climate in above three regions in Southern China. Based on a point evaluation and the Monte Carlo model, eggs and chicken tissues contributed a small amount to the mycotoxin intake for children, adults, and elderly adults (Wang et al., 2018). Our survey showed that the residues of $\mathrm{FB}$ and $\mathrm{DON}$ were much higher than the above reports. The different levels of mycotoxins in eggs might be associated with test methods and sampling seasons. Our samples were collected in the hot season and determined using a commercial ELISA kit. Low-mycotoxin residues were determined in the cold season based on our survey (data not shown). As children are sensitive to mycotoxin levels, it is worthwhile to pay attention to egg security during the hot season, along with egg storage methods.

In our study, a dose-dependent hatching rate was detected in the $\mathrm{FB}_{1}$ groups, the $\mathrm{FB}_{2}$ groups, and the $\mathrm{FB}_{1}+\mathrm{DON}$ groups, suggesting a high risk to the developing embryos of breeder eggs. More importantly, both FB1 and DON via egg residuals yielded a harmful syngenetic effect on chicken progeny, threatening the sustainable poultry industry. Although FB contaminations are commonly documented in animal feed and feedstuff, the association between FB mycotoxins and hatching rate or gizzard ulcerations has not yet been thoroughly investigated. There are 28 known important $\mathrm{FB}$ analogy, among which $\mathrm{FB}_{1}$ is the most prominent, followed by $\mathrm{FB}_{2}$ and $\mathrm{FB}_{3}$. $\mathrm{FB}_{1}$ is known as a cancer promoter and plays a significant role in carcinogenesis in humans and immunosuppression in birds. Hatchability was adversely affected by the presence of DON in Lohmann brown (LB) hens' diet, while the hatchability of Lohmann LSL chicks was significantly higher than that of LB chicks. Moreover, the spleen relative weight and gizzard relative weight were significantly decreased in the hatching chicks postfeeding of the contaminated diets to the hens compared with the control group, indicating a negative impact of DON in LB hens' diet on fertility and hatchability (Ebrahem et al., 2014). In a previous study, breeder eggs were injected with serial doses of OTA before incubation, and the crown-to-rump length, optic cups, and eye lens diameters were reduced significantly. More interestingly, neural tube closure defects were higher in the OTA-treated embryos. Teratogenic defects and embryonic mortalities were higher in the groups administered high doses of OTA (Zahoor Ul et al., 2011). Prior to incubation, $\mathrm{AFB}_{1}$ was injected into the air space of the breeder eggs. Poor embryonic development of the tibial growth plate and skeletal disorders were observed in the hatched broilers during growth (Oznurlu et al., 2010).

Regarding gizzard ulcerations, the epithelium layer of the innermost mucosa is of crucial importance for intestinal barrier functioning. Generally, $\mathrm{FB}_{1}$ increases intestinal cell apoptosis, reducing the intestinal barrier and causing immune dysfunction (Gulbahce Mutlu et al., 2018). In the present study, an increase of gizzard ulcerations and hemorrhagic lesions in the lungs were observed in the synergetic high $\mathrm{FB}_{1}+\mathrm{DON}$ group, the moderate dose of the $\mathrm{FB}_{1}+\mathrm{DON}$ group, the high dose of the $\mathrm{FB}_{1}$ group, and the moderate dose of the $\mathrm{FB}_{1}$ group, as compared to the other groups. The above data demonstrated that $\mathrm{FB}_{1}$ mycotoxins have a positive effect on gizzard ulceration and lung inflammation. The synergetic combinations of FB1 and DON exacerbated gizzard ulcerations and hatching rate of chicken progenies. A possible mechanism might be associated with the accumulation of two toxins in a long term. Broiler chicks fed $\mathrm{FB}_{1}$ contaminated feed showed an altered sphingosine/sphingosine ratio, a biomarker of the $\mathrm{FB}$ effect due to disruption of sphingolipid metabolism. $\mathrm{FB}_{1}$ shares the same structure as cellular sphingolipids, and sphingolipids are responsible for neurological and immunological diseases, as well as cancer (Grenier et al., 2015). Secondly, $\mathrm{FB}_{1}$ altered the integrity of the intestinal barrier and promoted translocation of bacteria by suppressing the tight junction protein expression level. On other hand, DON was found to induce necrotic lesions in the GIT by increasing intestinal permeability and nutrition malabsorption 
(Liew and Mohd-Redzwan, 2018). In the present study, the combination of $\mathrm{FB}_{1}$ and $\mathrm{DON}$ induced significant gizzard ulcerations in a dose-dependent manner, indicating synergic effects of the two mycotoxin contaminations in breeder eggs. The GIT is responsible for feed ingestion, digestion, nutrient absorption, and the immune response in newborn chickens. A more comprehensive strategy is urgently required to maintain chicken health and food sustainability.

\section{CONCLUSION}

Our investigation attempted to highlight how feed-borne mycotoxins were transferred to breeder eggs by checking mycotoxin residues in yolk and albumen samples. Using an embryonated egg model, $\mathrm{FB}_{1}$ or the combination of $\mathrm{FB}_{1}$ with DON contributed to poor hatching rate and early inflammations of the gizzard ulcerations and hemorrhagic lungs, leading to higher mortality of the progeny of breeder hens and threatening consumable egg safety. Regardless, more studies are needed to elucidate the interaction between mycotoxins and the development of the embryonated chickens, and the implication of such interactions for mycotoxicosis prevention measures.

\section{DATA AVAILABILITY STATEMENT}

The original contributions presented in the study are included in the article/supplementary material, further inquiries can be directed to the corresponding author.

\section{REFERENCES}

Agriopoulou, S., Stamatelopoulou, E., and Varzakas, T. (2020). Advances in Occurrence, Importance, and Mycotoxin Control Strategies: prevention and Detoxification in Foods. Foods 9:137. doi: 10.3390/foods9020137

Antonissen, G., Croubels, S., Pasmans, F., Ducatelle, R., Eeckhaut, V., Devreese, M., et al. (2015). Fumonisins affect the intestinal microbial homeostasis in broiler chickens, predisposing to necrotic enteritis. Vet. Res. 46:98. doi: 10.1186/ s13567-015-0234-8

Atiqul Haque, M. D., Zonghui Zuo, H. Q., Khan, A., Siddique, N., and He, C. (2020). Pathogenicity of feed-borne Bacillus cereus and its implication on food safety. Agrobiol. Records 3, 1-16.

Broom, L. (2015). Mycotoxins and the intestine. Anim. Nutr. 1, 262-265. doi: 10.1016/j.aninu.2015.11.001

Chu, J., Zhang, Q., Zuo, Z., El-Ashram, S., Guo, Y., Zhao, P., et al. (2017). Coinfection of Chlamydia psittaci with H9N2, ORT and Aspergillus fumigatus contributes to severe pneumonia and high mortality in SPF chickens. Sci. Rep. 7:13997. doi: 10.1038/s41598-017-14519-1

Cordeiro, F., Baer, I., Robouch, P., Emteborg, H., Can, S. Z., Krata, A., et al. (2013). Setting maximum limits for trace elements in baby food in European legislation: the outcome of International Measurement Evaluation Programme(R)-33. Food Addit. Contam. Part A Chem. Anal. Control Expo. Risk Assess. 30, 678-686. doi: 10.1080/19440049.2013.775605

Dassi, M., Souto, N. S., Braga, A. C. M., Freitas, M. L., Vasconcelos, C., Oliveira, M. S., et al. (2018). Effects of repeated fumonisin B1 exposure on markers of oxidative stress in liver, kidneys, and lungs of C57BL/6 mice. J. Environ. Sci. Health B 53, 840-845. doi: 10.1080/03601234.2018.1505 258

Ebrahem, M., Kersten, S., Valenta, H., Breves, G., Beineke, A., Hermeyer, K., et al. (2014). Effects of feeding deoxynivalenol (DON)-contaminated wheat to laying hens and roosters of different genetic background on the reproductive

\section{ETHICS STATEMENT}

The animal study was reviewed and approved by the China Agricultural University.

\section{AUTHOR CONTRIBUTIONS}

$\mathrm{CH}$ : conceptualization and methodology. YW: data curation and writing-original draft. QL, QS, XL, YW, and HQ: investigation. QS and $\mathrm{MH}$ : project administration. YW and QF: resources. $\mathrm{CH}$ and QF: writing-review and editing. All authors contributed to the article and approved the submitted version.

\section{FUNDING}

This work was supported by the Taishan Scholar Foundation of Shandong Province under Grant No. ts201511084 and the Yuandou Industry Leading Talent of Shandong Province.

\section{ACKNOWLEDGMENTS}

Kun Dong and Hongguang Li helped our team to collect poultry diets and breeder eggs during the survey. Also, we are grateful for Jianlong Liu who provided technical service for sample detection.

performance and health of the newly hatched chicks. Mycotoxin Res. 30, 131-140. doi: 10.1007/s12550-014-0197-z

Willems, E., Decuypere, E., Buyse, J., and Everaert, N. (2014). Importance of albumen during embryonic development in avian species, with emphasis on domestic chicken. World Poult. Sci. J. 70, 503-507.

Grenier, B., Schwartz-Zimmermann, H. E., Caha, S., Moll, W. D., Schatzmayr, G., and Applegate, T. J. (2015). Dose-dependent effects on sphingoid bases and cytokines in chickens fed diets prepared with fusarium verticillioides culture material containing fumonisins. Toxins 7, 1253-1272. doi: 10.3390/ toxins 7041253

Gulbahce Mutlu, E., Arslan, E., Oznurlu, Y., and Ozparlak, H. (2018). The effects of aflatoxin $\mathrm{B} 1$ on growth hormone regulated gene-1 and interaction between DNA and aflatoxin B1 in broiler chickens during hatching. Biotech. Histochem. 93, 463-470. doi: 10.1080/10520295.2018.1454986

Henry, M. H., and Wyatt, R. D. (2001). The toxicity of fumonisin B1, B2, and B3, individually and in combination, in chicken embryos. Poult. Sci. 80, 401-407. doi: $10.1093 / \mathrm{ps} / 80.4 .401$

Khan, W. A., Khan, M. Z., Khan, A., Ul Hassan, Z., and Saleemi, M. K. (2014). Potential for amelioration of aflatoxin B1-induced immunotoxic effects in progeny of White Leghorn breeder hens co-exposed to vitamin E. J. Immunotoxicol. 11, 116-125. doi: 10.3109/1547691X.2013.804134

Lee, J. T., Jessen, K. A., Beltran, R., Starkl, V., Schatzmayr, G., Borutova, R., et al. (2012). Effects of mycotoxin-contaminated diets and deactivating compound in laying hens: 2. effects on white shell egg quality and characteristics. Poult. Sci. 91, 2096-2104. doi: 10.3382/ps.2012-02137

Liew, W. P., and Mohd-Redzwan, S. (2018). Mycotoxin: its Impact on Gut Health and Microbiota. Front. Cell. Infect. Microbiol. 8:60. doi: 10.3389/fcimb.2018. 00060

Manarolla, G., Pisoni, G., Moroni, P., Gallazzi, D., Sironi, G., and Rampin, T. (2009). Adenoviral gizzard erosions in Italian chicken flocks. Vet. Rec. 164, 754-756. doi: 10.1136/vr.164.24.754 
Oznurlu, Y., Celik, I., Telatar, T., and Sur, E. (2010). Histochemical and histological evaluations of the effects of high incubation temperature on embryonic development of thymus and bursa of Fabricius in broiler chickens. Br. Poult. Sci. 51, 43-51. doi: 10.1080/00071660903575558

Prelusky, D. B., Hamilton, R. M., and Trenholm, H. L. (1989). Transmission of residues to eggs following long-term administration of $14 \mathrm{C}$-labelled deoxynivalenol to laying hens. Poult. Sci. 68, 744-748. doi: 10.3382/ps.0680744

Spanic, V., Zdunic, Z., Drezner, G., and Sarkanj, B. (2019). The Pressure of Fusarium Disease and Its Relation with Mycotoxins in The Wheat Grain and Malt. Toxins 11:198. doi: 10.3390/toxins11040198

Sypecka, Z., Kelly, M., and Brereton, P. (2004). Deoxynivalenol and zearalenone residues in eggs of laying hens fed with a naturally contaminated diet: effects on egg production and estimation of transmission rates from feed to eggs. J. Agric. Food Chem. 52, 5463-5471. doi: 10.1021/jf040039d

Tangni, E. K., Waegeneers, N., Van Overmeire, I., Goeyens, L., and Pussemier, L. (2009). Mycotoxin analyses in some home produced eggs in Belgium reveal small contribution to the total daily intake. Sci. Total Environ. 407, 4411-4418. doi: 10.1016/j.scitotenv.2008.10.060

Vesely, D., Vesela, D., and Jelinek, R. (1982). Nineteen mycotoxins tested on chicken embryos. Toxicol. Lett. 13, 239-245. doi: 10.1016/0378-4274(82)902 18-1

Wang, L., Zhang, Q., Yan, Z., Tan, Y., Zhu, R., Yu, D., et al. (2018). Occurrence and Quantitative Risk Assessment of Twelve Mycotoxins in Eggs and Chicken Tissues in China. Toxins 10:477. doi: 10.3390/toxins10110477

Warth, B., Parich, A., Atehnkeng, J., Bandyopadhyay, R., Schuhmacher, R., Sulyok, M., et al. (2012). Quantitation of mycotoxins in food and feed from Burkina
Faso and Mozambique using a modern LC-MS/MS multitoxin method. J. Agric. Food Chem. 60, 9352-9363. doi: 10.1021/jf302003n

Zahoor Ul, H., Khan, M. Z., Khan, A., Javed, I., and Saleemi, M. K. (2011). Immunological status of the progeny of breeder hens kept on ochratoxin A (OTA)-contaminated feed. J. Immunotoxicol. 8, 122-130. doi: 10.3109/ 1547691X.2010.547886

Zhang, Q., Zuo, Z., Guo, Y., Zhang, T., Han, Z., Huang, S., et al. (2019). Contaminated feed-borne Bacillus cereus aggravates respiratory distress post avian influenza virus H9N2 infection by inducing pneumonia. Sci. Rep. 9:7231. doi: 10.1038/s41598-019-43660-2

Zuo, Z., Li, Q., Guo, Y., Li, X., Huang, S., Hegemann, J. H., et al. (2020). Feedborne Bacillus cereus exacerbates respiratory distress in chickens infected with Chlamydia psittaci by inducing haemorrhagic pneumonia. Avian Pathol. 49, 251-260. doi: 10.1080/03079457.2020.1716940

Conflict of Interest: The authors declare that the research was conducted in the absence of any commercial or financial relationships that could be construed as a potential conflict of interest.

Copyright (C) 2021 Wang, Quan, Li, Li, Haque, Shi, Fu and He. This is an openaccess article distributed under the terms of the Creative Commons Attribution License (CC BY). The use, distribution or reproduction in other forums is permitted, provided the original author(s) and the copyright owner(s) are credited and that the original publication in this journal is cited, in accordance with accepted academic practice. No use, distribution or reproduction is permitted which does not comply with these terms. 